\title{
Chlamydial infection of the urogenital tract in promiscuous and non-promiscuous women
}

\author{
J. M. G. WOOLFITT AND L. WATT \\ From the Special Clinic, Royal Infirmary, Manchester
}

SUMMARY Chlamydia trachomatis infection of the urogenital tract of women presumed to be non-promiscuous, and in presumably promiscuous women attending a Special Clinic in Manchester was studied. Two hundred female members of hospital staff, who formed the non-promiscuous group, were found to have a $1 \%$ incidence of chlamydial infection. This compared with an incidence of $26 \%$ among 200 women attending the clinic. Among the clinic patients, chlamydial infection was significantly linked with the presence of gonorrhoea, mixed infections, and other sexually transmissible diseases-such as, trichomoniasis. No correlation could be made between the incidence of chlamydial infection and the use of oral contraceptives.

\section{Introduction}

Few investigations to determine the incidence of infection with Chlamydia trachomatis in the urogenital tract of women who are presumed not to be promiscuous have been reported. Investigation in a Family Planning Clinic in Bristol revealed chlamydial infection in only $3 \%$ of 63 women studied (Hilton et al., 1974). In another study chlamydial infection was detected in only $3.9 \%$ of a selected group of 77 women who attended a venereal disease clinic but were found to be free from evidence of sexually transmissible disease (STD), or as far as was known, contact with such disease (Burns et al., 1975).

Reported chlamydial isolation rates in women presumably exposed to a wider variety of sexual contact and attending venereal disease clinics varied from $11 \%$ of 638 patients (Burns et al., 1975) to $31 \%$ of 279 (Hilton et al., 1974).

\section{Material and methods}

The current study on two groups was made between September 1973 and September 1974 in Manchester Royal Infirmary. The first group comprised 200 women from the staff of Manchester Royal Infirmary who volunteered to have specimens taken for chlamydial isolation at the same time as specimens for cervical cytology. The second group consisted of

Address for reprints Dr L. Watt, Consultant Venereologist, Manchester Royal Infirmary, Oxford Road, Manchester M13 9WL

Received for publication 23 August 1976
200 unselected women attending the Special Clinic at Manchester Royal Infirmary. The mean ages of the two groups were 28.86 and 23.71 years, respectively. It is assumed that the patients who attended the Special Clinic had either themselves been promiscuous or had been exposed to the possible results of promiscuity, while the staff members comprised a non-promiscuous group.

The women attending the Special Clinic were consecutive patients, presenting for the first time. All were examined by the same clinician (LW), who also took specimens for investigation. Specimens from the hospital staff were taken by three clinicians all of whom had been instructed in the technique.

All patients attending the Special Clinic were examined for evidence of sexually transmitted disease (STD). Smears and cultures were examined for Neisseria gonorrhoeae and also for Trichomonas vaginalis and Candida albicans, under phase contrast microscopy. Smears were made for cervical cytology from both groups.

Material for chlamydial culture was collected from the cervical canal in all patients, and from the urethral meatus in patients attending the Special Clinic. Plain cotton albumen-coated swabs were used. These were placed immediately into plastic capsules containing sugar-phosphate transport medium (2SP) with $3 \%$ fetal calf serum and antibiotics (Darougar et al., 1972). The specimens were snap frozen and stored at a temperature of $-180^{\circ} \mathrm{C}$.

Monolayers of irradiated McCoy cells were prepared on circular cover slips at the base of sterile flat-bottomed tubes. Specimens were inoculated 
into two tubes using the simplified one passage technique (Darougar et al., 1971). The tubes were centrifuged at $\times 10000 \mathrm{~g}$ for one hour at $30^{\circ} \mathrm{C}$, and subsequently incubated at $35^{\circ} \mathrm{C}$ for 60 hours. Using dark field illumination, Giesma-stained monolayers were then examined for the presence of chlamydial inclusions using a $\times 10$ objective and $\times 10$ eyepiece.

\section{Results}

INCIDENCE OF CHLAMYDIAL INFECTION Table 1 shows the incidence of chlamydial infection in the study groups. Infection was detected in only $1 \%$ of hospital staff compared with $26.5 \%$ of the group attending the Special Clinic.

Table 1 Incidence of C. trachomatis infections

\begin{tabular}{llcc}
\hline & & \multicolumn{2}{c}{ Chlamydia-positive } \\
\cline { 3 - 4 } Women & No. & No. & $\%$ \\
\hline Hospital staff & 200 & 2 & 1 \\
Clinic patients & 200 & 53 & 26.5 \\
\hline
\end{tabular}

SITE OF INFECTION

In females attending the Special Clinic Chlamydia were isolated more often from the cervix than from the urethra (Table 2). Thirty-two patients had detectable chlamydial infections of the cervix, 16 had both cervical and urethral infection, and five had urethral infection only. A total of 21 patients $(10.5 \%)$ had urethral infection.

Table 2 Site of infection of 200 clinic patients

\begin{tabular}{lll}
\hline Site & No. infected & Chlamydia-positive $\%$ \\
\hline Cervix only & 32 & 16 \\
Cervix and urethra & 16 & 8 \\
Urethra only & 5 & $2 \cdot 5$ \\
\hline
\end{tabular}

EFFECT OF OTHER PATHOGENS

Table 3 shows that patients with gonorrhoea, trichomoniasis, and mixed infections had a significantly high incidence of chlamydial infection. Patients who had genital inflammation due to a

Table 3 Association of chlamydial infections with other STD in clinic patients

\begin{tabular}{lllllc}
\hline $\begin{array}{l}\text { Female } \\
\text { patients }\end{array}$ & $\begin{array}{l}\text { No } \\
\text { STD }\end{array}$ & $\begin{array}{l}\text { Gonorrhoea } \\
\text { only }\end{array}$ & $\begin{array}{l}\text { Trichomoniasis } \\
\text { only }\end{array}$ & $\begin{array}{l}\text { Other } \\
\text { infections }\end{array}$ & $\begin{array}{l}\text { Mixed } \\
\text { infections }\end{array}$ \\
\hline $\begin{array}{l}\text { No. } \\
\text { Chlamydia- }\end{array}$ & 74 & 32 & 37 & 40 & 17 \\
$\begin{array}{l}\text { positive } \\
\text { (No.) }\end{array}$ & 10 & 13 & 13 & & \\
$\%$ & 13 & 40 & 35 & 9 & 8 \\
\hline
\end{tabular}

single infective agent-that is, gonorrhoea, trichomoniasis, candidiasis, herpes genitalis, or warts were classified as having other infections. Those with genital lesions resulting from more than one infective agent, were classified as having mixed infections.

Chlamydia were isolated in $13 \%$ of patients with no other detectable STD compared with $40 \%$ of those with gonorrhoea $\left(\chi_{1}^{2}=9.96,0.005>\mathrm{P}>0.001\right)$. There was also a significantly high incidence of Chlamydia (35\%) among patients with trichomoniasis $\left(\chi_{1}^{2}=7.02,0.01>P>0.005\right)$, and of $47 \%$ among those with mixed infections $\left(\chi_{1}^{2}=9 \cdot 80\right.$, $0.005>P>0.001)$. This suggests that Chlamydia are associated with the presence of other infections.

\section{CLINICAL SIGNS}

Clinical signs of vaginitis, urethritis, cervicitis, and cervical erosion could not be related to the incidence of chlamydial infection among clinic patients. Chlamydia were harboured by $20 \%$ of 30 women, who showed no clinical signs.

\section{EFFECT OF ORAL CONTRACEPTIVES}

In the 154 patients in the clinic group for whom details were available, Chlamydia were isolated from $23(26.1 \%)$ of 88 patients not using oral contraceptives and from $17(25 \cdot 7 \%)$ of 66 who were. This difference is not statistically significant, and no correlation can be made between the use of oral contraceptives and the incidence of chlamydial infection.

\section{MARITAL STATUS}

Table 4 shows that there was a highly significant difference between the marital status of women in the two groups. In the clinic patients $23.5 \%$ were married compared with $45.5 \%$ of the staff group $\left(\chi_{1}^{2}=16 \cdot 87,<0.0005\right)$.

Table 4 Marital status of study groups

\begin{tabular}{llll}
\hline & Single & Married & Divorced \\
\hline Hospital staff & 109 & 91 & 0 \\
Clinic patients & 138 & 47 & 15 \\
\hline
\end{tabular}

\section{COUNTRY OF ORIGIN}

No details are available concerning the ethnic origin of any of the patients, but the country of birth of the clinic patients was recorded. Of the 173 clinic patients born in the United Kingdom, 43 were infected with Chlamydia $(24.8 \%)$. Fifteen were from the West Indies and of these seven had chlamydial infections $(46.6 \%)$. Of nine patients born in Canada and Europe, three had chlamydial infection, and no such infection was demonstrated 
in two women born in Asia and one woman born in Africa. There are no statistically significant differences in these results.

\section{Discussion}

The chlamydial isolation rate of $26 \%$ from clinic patients, $23.5 \%$ of whom were married, is comparable with the earlier results from a similar clinic in Bristol (Hilton et al., 1974). The $1 \%$ incidence of Chlamydia among the female hospital staff, $45 \cdot 5 \%$ of whom were married, is also comparable with the result from non-promiscuous women attending the Bristol Family Planning Clinic (Hilton et al., 1974).

Only cervical material was collected from the staff group. It is unlikely that many chlamydial infections of the female urethra remained undetected, since only $2.5 \%$ of the female clinic patients had chlamydial infection which was confined to the urethra.

Among clinic patients, the results show that Chlamydia are significantly linked with the presence of gonorrhoea, trichomoniasis, or mixed infections. The results are comparable with those of Oriel et al. (1974) who isolated the agent from $32 \%$ of women with gonorrhoea. Hilton et al. (1974) found that $62 \%$ of 40 women with gonorrhoea, and $59 \%$ of 22 with mixed infections had chlamydial infections. Recently, Burns et al. (1975) found Chlamydia in $43 \%$ of 32 women with gonorrhoea.

Of the 37 patients with trichomoniasis in this series $35 \%$ had a chlamydial infection, an incidence comparable with the $24 \%$ of a previous report from Bristol (Hilton et al., 1974), but higher than the $13 \%$ reported from London by Burns et al. (1975). In another London survey (Oriel et al., 1974), only one out of 25 women with trichomoniasis, harboured Chlamydia. These workers questioned why in contrast to gonorrhoea, trichomoniasis was associated only occasionally with chlamydial infection.
From these results, Chlamydia seem to be linked with trichomonal infections.

Oral contraceptives may exacerbate some genital disorders in women. A previous report showed that women taking oral contraceptives had a significantly higher incidence of chlamydial infection (Hilton et al., 1974). In the present study, no correlation could be made between the use of oral contraception and the incidence of Chlamydia. This is in agreement with the findings of other reports (Oriel et al., 1974; Burns et al., 1975).

If Chlamydia are considered as a potential cause of STD, the asymptomatic carrier state, which appears to exist in some females, assumes epidemiological importance. In this investigation, Chlamydia were isolated from $20 \%$ of asymptomatic females, indicating the possibility of a considerable potential reservoir of infection.

We wish to thank $\mathrm{Dr} M$. Bennett, community physician Manchester AHA(T) and her staff for their co-operation.

\section{References}

Burns, D. C. M., Darougar, S., Thin, R. N., Lothian, L., and Nicol, C. S. (1975). Isolation of Chlamydia from women attending a clinic for sexually transmitted disease. British Journal of Venereal Diseases, 51, 314-318.

Darougar, S., Jones, B. R., Kinnison, J. R., Vaughan-Jackson, J. D., and Dunlop, E. M. C. (1972). Chlamydial infection. Advances in the diagnostic isolation of Chlamydia, including TRIC agent, from the eye, genital tract, and rectum. British Journal of Venereal Diseases, 48, 416-420.

Darougar, S., Kinnison, J. R., and Jones, B. R. (1971). Chlamydial isolates from the rectum in association with chlamydial infection of the eye or genital tract. I. Laboratory aspects. In Trachoma and Related Disorders, pp. 501-506. Edited by R. L. Nichols. Excerpta Medica: Amsterdam and New York.

Hilton, A. L., Richmond, S. J., Milne, J. D., Hindley, F., and Clarke, S. K. R. (1974). Chlamydia A in the female genital tract. British Journal of Venereal Diseases, 50, 1-10.

Oriel, J. D., Powis, P. A., Reeve, P., Miller, A., and Nicol, C. S. (1974). Chlamydial infections of the cervix. British Journal of Venereal Diseases, 50, 11-16. 